Letter to the Editor

\title{
Stable microvascular angina: Instrumental evaluation of coronary microvascular dysfunction with coronary angiography and myocardial scintigraphy
}

\author{
Vincenzo Sucato *, Salvatore Evola, Giuseppina Novo, Enrico Bronte, Salvatore Novo \\ Division of Cardiology, Biomedical Department of Internal Medicine and Medical Specialties (DIBIMIS), University Hospital Paolo Giaccone, Palermo, Italy.
}

\section{A R T I C L E I N F O}

\section{Article history:}

Received 12 February 2013

Received in revised form 17 December 2013

Accepted 20 December 2013

Available online 2 January 2014

\section{Keywords:}

Microvascular angina

Chest pain

Coronary angiography

Microcirculation

Myocardial scintigraphy

Stable microvascular angina (SMVA) describes a framework in which episodes of angina are exclusively or predominantly related to exertion. During diagnostic investigation, physician notices findings compatible with myocardial ischemia, normal coronary arteries on angiography, absence of any other specific cardiac disease (e.g., variant angina, cardiomyopathy, and valvular disease) [1,2]. The aim of this study is to demonstrate that myocardial ischemia in patients without epicardial artery stenosis, evaluated with nuclear imaging, correlates with alterations of coronary microcirculation. These alterations were evaluated by angiography indices like TIMI Frame Count (TFC), Myocardial Blush Grade (MBG) and Total Myocardial Blush Score (TMBS).

We studied ninety-six patients who came to the emergency room for angina from January 2007 to May 2011. After that they were hospitalized to our institution. In this retrospective study, inclusion criteria are the presence of stable angina, the presence of positive stress test, and coronary arteries free from stenosis at coronary angiography. All patients underwent to myocardial scintigraphy and coronary angiography. Significantly, we excluded patients who had positive biomarkers for myocardial infarction when they arrived to the Emergency Room or during hospitalization. Besides, we considered neither those patients who had the mild increase after coronary angiography, nor those pa-

\footnotetext{
* Corresponding author at: Division of Cardiology, Biomedical Department of Internal Medicine and Medical Specialties (DIBIMIS), University Hospital Paolo Giaccone, Via De Vespro no. 129, 90127 Palermo, Italy. Tel.: + 391655 4301; fax: + 3916554304.

E-mail address: odisseos86@alice.it (V. Sucato).
}

tients who had ischemic heart disease in history. We divided our population into two groups: sixty-one patients (63.5\%) with positive scintigraphy, and thirty-five patients (36.4\%) with negative scintigraphy. Based on the angiographic images, we calculated TIMI Frame Count and Myocardial Blush Grade for each patient. The TIMI Frame Count was calculated according to three main coronary arteries (LAD = left anterior descendent artery; $C X=$ circumflex coronary artery; RCA = right coronary artery). The protocol described by Gibson et al was used. Furthermore, the longer LAD frame counts were corrected dividing by 1.7 to obtain the corrected TIMI Frame Count (CTFC). We did this to make them comparable with those reported in literature $[3,4]$. Using the same calculation principle, we calculated the Myocardial Blush Grade carefully following the protocol described by Gibson et al. Besides, we used a new index proposed by Yusuf et al., the Total Myocardial Blush Score (TMBS). We obtained it summing up the Myocardial Blush Grade of each coronary area [5]. On the basis of the experience by Yusuf et al., we evaluated another index, the Total TIMI Frame Count (TTFC). We obtained it from the sum of the TFCs of the three coronary arteries. We also evaluated whether the results obtained through TTFC were comparable with the other indices we mentioned. The aim was to use this new index for a more comprehensive view of the coronary microcirculation.

Descriptive statistics were presented as percentages for categorical variables and as mean values \pm standard deviation (SD) for continuous data. We used a two-tailed t-student test to determine the statistical significance of difference between continuous variables. We made a one-way analysis of variance (ANOVA) test to show a normal distribution between continuous variables. A p-value $<0.05$ was considered statistically significant. Statistical analysis was performed using the Med Calc Program.

The population we examined included ninety-six patients. These patients were subjected to coronary angiography study. It detected epicardial coronary arteries free from stenosis and myocardial scintigraphy. They underwent the examination after having stable angina of considerable intensity at rest and a positive stress test. Sixty-one patients had positive scintigraphy (63\%) and thirty-five patients (36\%) had negative scintigraphy. We studied these two subgroups to determine which patients had a greater involvement of microcirculation. Our analysis started by studying the TIMI Frame Count, the flow coronary index on the three coronary arteries. We showed that patients with positive scintigraphy had a longer TFC of three major coronary arteries (TFC LAD, $45.7 \pm 12.5$; TFC RCA, $25.6 \pm 6.5$; TFC CX, $24 \pm 5.4$ ), than negative 
Table 1

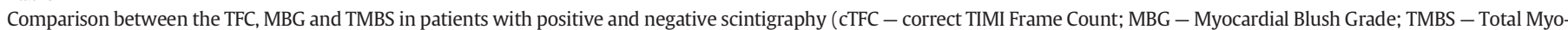
cardial Blush Score; TTFC - Total TIMI Frame Count). (LAD = left anterior descendent artery; CX = circumflex coronary artery; RCA = right coronary artery).

\begin{tabular}{|c|c|c|c|}
\hline & Patients with positive scintigrapgy (61) & Patients with negative scintigraphy (35) & p-Value \\
\hline MBG LAD & $2.2( \pm 0.4)$ & $2.4( \pm 0.5)$ & 0.03 \\
\hline MBG RCA & $2.2( \pm 0.4)$ & $2.3( \pm 0.47)$ & NS \\
\hline MBG CX & $2.1( \pm 0.36)$ & $2.3( \pm 0.4)$ & 0.04 \\
\hline TMBS & $5.6( \pm 1.06)$ & $7( \pm 1.16)$ & 0.003 \\
\hline CTFC LAD & $45.7( \pm 12.5)$ & $41( \pm 11.6)$ & NS \\
\hline TFC RCA & $25.6( \pm 6.5)$ & $25.1( \pm 6.3)$ & NS \\
\hline TFC CX & $24( \pm 5.4)$ & $21( \pm 4.7)$ & 0.05 \\
\hline TTFF & $106( \pm 13.2)$ & $101.7( \pm 12.1)$ & 0.02 \\
\hline
\end{tabular}

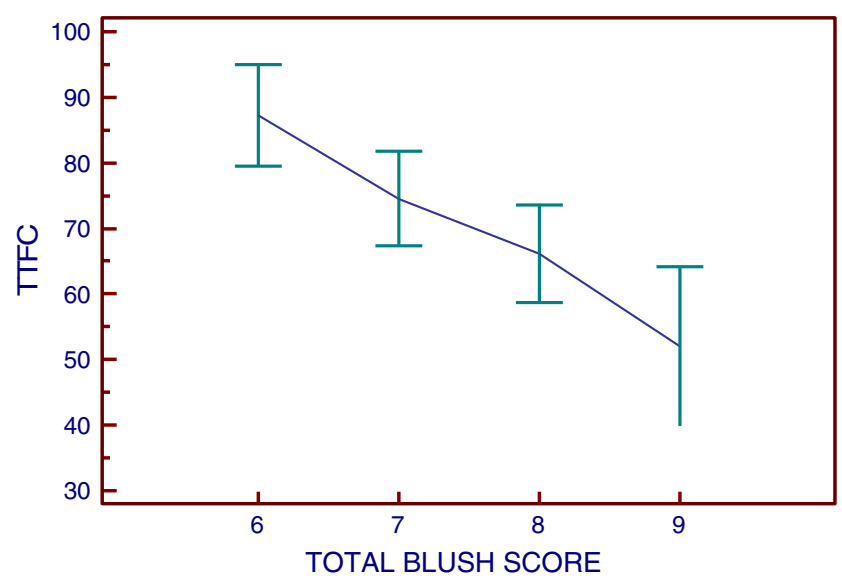

Fig. 1. Connection between the Total Myocardial Blush Score (TMBS) and the Total TIMI Frame Count (TTFC) in patients with positive scintigraphy. When TMBS, index of a better perfusion of the microcirculation, increases there is a decrease of TTFC, low flow velocity index.

scintigraphy patients. This indicates slow flow in positive scintigraphy coronary microcirculation (Table 1 ). Then we studied coronary microcirculation perfusion through Myocardial Blush Grade. We enlightened a lower MBG on the three coronary arteries (MBG LAD, $2.2 \pm 0.4$; MBG RCA, $2.2 \pm 0.4$; MBG CX, $2.1 \pm 0.36$ ) in patients with positive scintigraphy than patients with negative scintigraphy. There was a good statistical significance (Table 1). TMBS was lower in patients with positive scintigraphy. It had a good statistical significance too (TMBS, $5.6 \pm 1.06)(p$-value $=0.003$ ) (Table 1$)$. Finally, the index we suggested, the TTFC showed a greater value in patients with positive scintigraphy than patients with negative scintigraphy (TTFC, $106 \pm 13.2$ ) $(\mathrm{p}$-value $=0.02)($ Table 1$)$. So, we analyzed the connection between the Total Myocardial Blush Score and the Total TIMI Frame Count. We found, through a one way analysis of variance (ANOVA) test, an inverse proportionality. The increase of the TTFC, which highlight a slow flow of the contrast, goes hand in hand with the reduction of TMBS. This underlines its slow removal from the microvascular territory perfused by the same coronaries (Fig. 1). This correspondence highlights the relationship between the two indices and their capacity to detect the microvascular dysfunction. The results of scintigraphy, which is positive in 69.7\% patients, endorsed the hypothesis of a good connection between the study of the microcirculation realized through myocardial scintigraphy and coronary angiography analysis that we performed using the indices by Gibson and Yusuf. The myocardial scintigraphy showed myocardial perfusion abnormalities. The presence of perfusion abnormalities in absence of coronary macroangiopathy shows a link to microvascular abnormalities. On the other hand, the procedure identifies microcirculatory alterations. These alterations are part of a very early stage of the ischemic cascade, which is the redistribution of coronary blood flow.

The aim of this study was to compare myocardial scintigraphy to the indices by Gibson and Yusuf in coronary angiography in order to evaluate if there are analogies, studying patients affected by SMVA. Patients with positive coronary angiography, representing $69 \%$ of our population, had a TMBS minor than patients with positive scintigraphy $(p=0.002)$. In addition, the concurrence between the defect in scintigraphy and the defect in coronary angiography showed statistical significance. We showed this aspect from the comparison between the TFC of the damaged microvascular arteries in scintigraphy and the average of TFCs in the remaining healthy vessels in a certain patient $[6,7]$. From our work, we observed that the study of microcirculation, performed through myocardial scintigraphy and coronary angiography, showed a good connection between the two procedures [8]. This allows us to use a technique less invasive and reproducible such as myocardial scintigraphy as opposed to coronary angiography especially in the followup of patients affected by SMVA $[9,10]$. The use of indices in coronary angiography such as the TFC, MBG and TMBS is a useful parameter to evaluate coronary microvascular alterations. They have a good connection with scintigraphy. Finally, the TTFC is as well important, the new index we introduced. It has proved to be a good marker concordant with the results of the other indices.

\section{References}

[1] Lanza GA, Crea F. Primary coronary microvascular dysfunction: clinical presentation, pathophysiology, and management. Circulation 2010;121:2317-1325.

[2] Sucato V, Evola S, Novo G, Novo S. Diagnosis of coronary microvascualar dysfunction in diabetic patients with cardiac syndrome X: comparison by current methods. Recenti Prog Med 2013;104(2):63-8.

[3] Gibson CM, Cannon CP, Daley WL, et al. TIMI Frame Count: a quantitative method of assessing coronary artery flow. Circulation 1996;93:879-88.

[4] Atmaca Y, Ozdemir AO, Ozdol C, et al. Angiographic evaluation of myocardial perfusion in patients with syndrome X. Am J Cardiol 2005;96:803-5.

[5] Atmaca Y, Duzen V, Ozdol C, et al. Total blush score: a new index for the assessment of microvascular perfusion in idiopathic dilated cardiomyopathy. Coron Artery Dis 2008; $19: 181-5$

[6] Sari I, Soydinc S, Davutoglu V, Sezen Y, Aksoy M. Uncomplicated diabetes mellitus is equivalent for coronary artery disease: new support from novel angiographic myocardial perfusion-myocardial blush. Int J Cardiol 2008;127:262-5.

[7] Vesely MR, Dilsizian V. Microvascular angina: assessment of coronary blood flow, flow reserve, and metabolism. Curr Cardiol Rep 2011;13:151-8.

[8] Fragasso G, Chierchia SL, Arioli F, et al. Coronary slow-flow causing transient myocardial hypoperfusion in patients with cardiac syndrome X: long-term clinical and functional prognosis. Int J Cardiol 2009;2:137-44.

[9] Lamendola P, Lanza GA, Spinelli A, et al. Long-term prognosis of patients with cardiac syndrome X. Int J Cardiol 2010;140:197-9.

[10] Lanza GA, Camici PG, Galiuto L, et al. Methods to investigate coronary microvascular function in clinical practice. J Cardiovasc Med 2013;14:1-18. 